\title{
Auditory Abnormalities in Autism: Toward Functional Distinctions Among Findings
}

\author{
By Gabriella R. Kellerman, BA, Jin Fan, PhD, and Jack M. Gorman, MD
}

\section{Needs Assessment}

While a wave of new interest has brought a resurgence of research on auditory abnormalities in autism, functional distinctions among the many, varied findings amassed are lacking. This review provides a framework for synthesizing current findings for clinicians, researchers, and patients who stand to benefit from greater clarity as to their scientific and therapeutic significance.

\section{Learning Objectives}

At the end of this activity, the participant should be able to:

- Differentiate among the many varied auditory abnormalities that have been demonstrated in individuals with autism.

- List examples of both enhanced and impaired auditory perceptions among individuals with autism.

- Relate these deficits and enhancements to the three major symptom clusters of autism: linguistic deficits, repetitive behaviors, and social impairments.

- Understand the potential that studies in this field hold for testing the global versus local processing theory of autism.

\section{Target Audience}

Neurologists and psychiatrists

\section{Accreditation Statement}

Mount Sinai School of Medicine is accredited by the Accreditation Council for Continuing Medical Education to provide Continuing Medical Education for physicians.

Mount Sinai School of Medicine designates this educational activity for a maximum of 3.0 Category 1 credit(s) toward the AMA Physician's Recognition Award. Each physician should claim only those credits that he/she actually spent in the educational activity. Credits will be calculated by the MSSM OCME and provided for the journal upon completion of agenda.

It is the policy of Mount Sinai School of Medicine to ensure fair balance, independence, objectivity and scientific rigor in all its sponsored activities. All faculty participating in sponsored activities are expected to disclose to the audience any real or apparent discussion of unlabeled or investigational use of any commercial product or device not yet approved in the United States.

This activity has been peer-reviewed and approved by Eric Hollander, MD, professor of psychiatry, Mount Sinai School of Medicine. Review Date: July 26, 2005.

\section{To Receive Credit for This Activity}

Read this article, and the two CME-designated accompanying articles, reflect on the information presented, and then complete the CME quiz found on pages 757 and 758. To obtain credits, you should score $70 \%$ or better. Termination date: September 30, 2007. The estimated time to complete this activity is 3 hours.

\begin{abstract}
Recently, findings on a wide range of auditory abnormalities among individuals with autism have been reported. To date, functional distinctions among these varied findings are poorly established. Such distinctions should be of interest to clinicians and researchers alike given their potential therapeutic and experimental applications. This review suggests three general trends among these findings as a starting point for future analyses. First, studies of auditory perception of linguistic and social auditory stimuli among individuals with autism generally have found impaired perception versus normal controls. Such findings may correlate with impaired language and communication skills and social isolation observed among individuals with autism. Second, studies of auditory perception of pitch and music among individuals with autism generally have found
\end{abstract}

enhanced perception versus normal controls. These findings may correlate with the restrictive and highly focused behaviors observed among individuals with autism. Third, findings on the auditory perception of non-linguistic, nonmusical stimuli among autism patients resist any generalized conclusions. Ultimately, as some researchers have already suggested, the distinction between impaired global processing and enhanced local processing may prove useful in making sense of apparently discordant findings on auditory abnormalities among individuals with autism.

CNS Spectr. 2005;10(9):748-756

\section{INTRODUCTION}

Autism is a severe developmental disorder characterized by three clusters of symptoms: impaired social interaction, language and communication deficits,

Ms. Kellerman is a third-year medical student at Mount Sinai School of Medicine (MSSM) in New York City. Dr. Fan is an assistant professor in the Department of Psychiatry at MSSM. Dr. Gorman is the editor of CNS Spectrums and professor of psychiatry and professor of neuropsychiatry in the Department of Psychiatry at MSSM.

Disclosure: The authors do not have an affiliation with or financial interest in any organization that might pose a conflict of interest. This article was submitted on November 3, 2004, and accepted on June 1, 2005.

Acknowledgment: The authors would like to acknowledge Jesse Kellerman for his support of this project.

Please direct all correspondence to: Gabriella R. Kellerman, BA, 12 E. 97th St. \#4B, New York, NY 10029; Tel: 646-672-0085; E-mail: grosen@post.harvard.edu. 
and restricted repetitive and stereotyped behavioral patterns. ${ }^{1}$ Sensory and perceptual abnormalities have also long been noted among patients with autism and were once a focal point for the study and treatment of the disorder. However, for the last 20 years research has revolved around the aforementioned symptom clusters, with investigations centering on impairments in social cognition (eg, theory of mind), social communication, and affect. ${ }^{2}$ Yet, given a host of new research technologies and an apparent renewal of interest in the sensory abnormalities consistently associated with autism, research tides may again be turning. How and to what effect newer studies of sensory abnormalities may enhance our understanding of social cognitive and behavioral symptoms in autism patients remains to be seen.

Auditory processing in autism represents one area where the need for integration of newer data on sensory abnormalities with older cognitive and behavioral findings is becoming increasingly evident. A wide range of abnormalities in auditory perception in autistic patients has been described and, in the last decade, broadly elaborated with neuroimaging studies. Unfortunately, as findings on these auditory abnormalities proliferate, comparative and synthetic analyses of the extremely varied results have not kept pace. Only recently have studies on auditory abnormalities in autism have begun to tease out differences between the perception of noise and speech, for example, or between the perception of sounds and attentional orientation toward them.

Such distinctions are critical, given that particular abnormalities do seem to correlate with those three better-established and decidedly distinct symptom clusters of autism. For example, poor orientation to specific social auditory stimuli (eg, an individual's voice or hands clapping) has been related to impaired social skills., ${ }^{3,4}$ Links between deficiencies in linguistic perception and the impaired language skills observed in autism, meanwhile, have also been suggested.5,6 Enhanced reactivity to and discrimination of musical melodies and pitch may be connected to the third feature of autism: restricted and highly focused interests and behaviors. ${ }^{7-11}$

This review provides an overview of the major categories of data-primarily from imaging and event-related brain potentials (ERPs) studies-on auditory abnormalities in autism, with particular attention to distinctions between data on perception of linguistic and non-linguistic sounds.

A better understanding of distinctions among auditory abnormalities observed in autism will be of interest to clinicians and researchers in their efforts toward refined treatment and understanding of the etiologies of particular symptoms.

For researchers, greater clarity on the range of abnormalities described should be of use not only to those investigating auditory abnormalities directly but also to those planning auditory tasks for studies of other, specific disorder features. Investigations using an auditory task to assess social cognitive functions in patients with autism, for example, might come to very different conclusions depending on whether the study employed pure tones or verbal auditory stimuli to elicit the measured response.

For clinicians, correlations of auditory impairments with other, well-described symptoms may offer therapeutic promise in the form of language and music therapies that could alleviate correlated, nonauditory symptoms. ${ }^{12,13}$ One recent review ${ }^{13}$ proposed that treatments to improve auditory processing in autism patients may also significantly alleviate other wide-ranging symptoms, such as social, linguistic, and theory of mind deficiencies. All such findings are as yet equivocal and the American Academy of Pediatrics "does not support the claims of proponents that [Auditory Integration Training] treatments are efficacious." ${ }^{14}$ But evaluation of the potential efficacy of these therapies has been clouded by oversimplification of the underlying impairments. ${ }^{14}$ Greater clarity on the particular etiologies and clinical consequences of impairments may allow for treatments of not only the auditory symptoms but also of the disorder features with which they are correlated.

Therefore, improved discernment of distinctions among auditory abnormalities in autism should be of interest to clinicians, researchers, patients, and caretakers alike in their ongoing efforts to understand and treat this disorder. This review concludes with an examination of early attempts to organize the range of auditory abnormalities observed in autism into a coherent whole, one that may hold important implications for our understanding of sensory processing in autism more generally.

\section{IMPAIRED PERCEPTION OF SPEECH AND AUDITORY SOCIAL STIMULI}

Studies of the perception of linguistic or social auditory stimuli in patients with autism overwhelmingly have found that perception is impaired in a range of ways. This concurrence stands in stark contrast to the more muddled findings on non-linguistic, non-social auditory perception. Studies of speech and social stimulus perception have generally focused either on deficient orientation to linguistic or social stimuli; or on abnormal anatomi- 
cal localization of speech perception. This section examines those two major lines of current investigation, to the possible exclusion of other speech perception studies outside of such categories.

Interest in hearing as the basis for orientational attention in infants and children dates back to at least 1976. ${ }^{15}$ Among the recent research examining deficiencies in attentional orienting to social sounds and speech in autism patients, Dawson and colleagues ${ }^{3}$ determined that autism patients do not orient to either speech or non-linguistic social stimuli as well as they do to non-social sounds. The researchers videotaped autistic children's visual-orienting responses to both social sounds (hearing their name called or to the sound of hands clapping) and non-social sounds (a rattle or a jack-in-the-box). Compared with control children and to children with Down syndrome, children with autism severely failed to visually orient to social stimuli. While some orienting impairments to non-social stimuli were also observed, they were far-less pronounced than impairments in orienting toward social stimuli. This study also found that impairments in attention sharing in patients with autism correlated with failed orientation to social stimuli but not with the smaller deficits observed in orientation to non-linguistic stimuli. Dawson and colleagues $^{3}$ proposed that this exclusive correlation (between attention-sharing deficits and deficient orientation to social stimuli) suggests a link between the social and attentional deficits observed in autism patients. A recent follow-up study by Dawson and colleagues ${ }^{16}$ replicated and confirmed these findings.

Other studies of impaired orientation to auditory social stimuli $i^{4-6}$ have focused exclusively on speech or linguistic stimuli, as opposed to non-linguistic stimuli. Their findings are, thus, primarily relevant to the language and communication deficit symptom cluster in autism and secondarily related to social impairment symptoms. Klin ${ }^{4}$ has shown autistic children's preference for non-linguistic background noise over that of their own mothers' voices. The children's responses were monitored in their homes by a computerized device. Compared with the children with autism, $\mathrm{Klin}{ }^{4}$ found that both normal and mentally retarded children strongly preferred their mother's voices to non-linguistic background noise.

One of the most important findings on speech processing, as far as the present review is concerned, came from Ceponiene and colleagues. ${ }^{5}$ In an ERP study, they examined sensory and attentional processing of simple sounds, complex sounds, and linguistic (vowel) sounds in high-functioning autistic children versus those of controls. Attentional processing was assessed by the subject's ERP response to alterations in the frequency of a specific category of sound (oddball task). They found that sensory processing in autistic children was normal and not affected by either the complexity of the sound processed or by its linguistic quality. That is, autistic children sensed all sounds, speech and non-speech, simple and complex alike, on par with the control children. In the attentional task, autistic children showed a deficit in attentional processing of phonemes. However, they exhibited normal attentional processing of simple and complex non-linguistic sounds alike. Thus, while autistic children's ERPs in response to changes in non-linguistic sounds did not differ from those of the control group, their ERPs in response to changes in linguistic sounds differed significantly, lacking the P3a wave characteristic of attentional orienting. On the basis of this observed dissociation between autistic children's normal sensory and impaired attentional, processing of linguistic sound, Ceponiene and colleagues ${ }^{5}$ proposed a speech-specific, post-sensory auditory impairment that may help explain the language deficits observed in autism.

Kemner and colleagues' ${ }^{\prime 6} 1995$ study may provide support for this post-sensory linguistic-processing deficit hypothesis. They studied ERPs in oddball tasks using linguistic (phoneme) standard and deviant sounds and a non-linguistic novel sound (standard=oy, deviant=ay, novel=brzzz), comparing imaging results of children with autism, attentiondeficit/hyperactivity disorder and dyslexic children with the results of normal controls. Confirming the results of two earlier studies by Courchesne and colleagues ${ }^{17}$ and Courchesne and colleagues, ${ }^{18}$ Kemner and colleagues ${ }^{6}$ found a decreased $\mathrm{A} / \mathrm{Pc} z / 300$ wave in autistic children's discrimination of standard versus deviant sounds relative to the same wave among the other three groups. This wave appears to be related to the P3a wave that was examined by Ceponiene and colleagues, ${ }^{5}$ although that connection is not certain. Kemner and colleagues ${ }^{6}$ also noted an increase in the occipital P3a wave in active oddball tasks as opposed to passive tasks among autism patients only (in the active task, subjects had to count the number of deviant sounds they heard), suggesting atypical occipital processing of auditory sounds in autism patients. Furthermore, Kemner and colleagues ${ }^{6}$ suggested that these finding may be related to increased difficulty with active auditory processing among individuals with autism, which induces the harnessing of different brain regions than are typically involved in the task. With respect to the question of why the region harnessed should be the occipital 
cortex, the researchers suggested a link to studies showing impaired facial recognition among autism patients. Consequent underutilization of occipital brain regions for visual processing, they proposed, may explain the availability of this region for appropriation for use in auditory processing.

Overall, these studies suggest significant impairments of attentional orienting toward linguistic, as opposed to non-linguistic, and toward social, as opposed to non-social, stimuli. These findings correlate well with both the social and linguistic impairments observed in individuals with autism.

A second important focus of studies of abnormalities in speech processing among autism patients has been the atypical localization of that processing. While much attention in autism neuroimaging has centered upon this sort of functional localization, and a range of abnormal localizations have been described, much work remains to be done on localizing speech perception. A functional magnetic resonance imaging study by Gervais and colleagues ${ }^{19}$ compared the response to vocal and non-vocal sounds of autism patients with those of control subjects. While the control group showed significantly greater activation of the superior temporal sulcus in response to vocal, as opposed to non-vocal, sounds, four of the five individuals with autism had the same superior temporal sulcus activation in response to vocal and non-vocal sounds. Gervais and colleagues ${ }^{19}$ also found a selective recall deficit for the voice, as opposed to non-voice, stimuli among individuals with autism. They proposed that these selective deficits for perception of and attention to voice data may help explain the impaired social interaction observed among individuals with autism. ${ }^{19}$

Several other localization studies of speech perception among individuals with autism have focused on atypical patterns of hemispheric dominance. A positron emission tomography study by Muller and colleague ${ }^{20}$ compared the functional localization of linguistic and non-linguistic auditory perception in five autistic adults with that in five control subjects. Among autistic adults, the group found reversed hemispheric dominance in response to verbal auditory stimuli; decreased auditory cortex activity during auditory stimulation of all types; and reduced cerebellar activity during nonverbal auditory perception. The latter two results are discussed later, as only the first (reversed hemispheric dominance) relates to speech specifically. The implications of the reversal in hemispheric dominance are unclear and the small subject pool further obfuscates the significance of this study results.
Stronger evidence for reversed dominance finding has come from Boddaert and colleagues. ${ }^{21}$ They have described abnormal cortical activation in autism patients during perception of "speech-like sounds." Such stimuli resemble consonants and vowels, and bilaterally activate the lateral belt of the cerebral cortex, known to be involved in early acoustic processing of speech. ${ }^{22,23}$ But because these sounds are never consciously recognized as speech-rather, they are said to sound electronic - their auditory processing in these areas may be considered both speech-specific and pre-linguistic. In keeping with the reversed dominance findings, ${ }^{20}$ Boddaert and colleagues ${ }^{21}$ found significantly greater activation in the right middle front gyrus of the temporal lobe than in the left among autistic patients, while the control group exhibited greater activation in the left gyrus. Because the stimuli were pre-linguistic, the results bear the important implication that the correlation between language deficits in autism and abnormal processing of speech sounds may be causal in one direction only. That is, based on these results, impaired language development seems not to be causing impaired linguistic perception. Rather, this pre-linguistic, but speech-specific, processing abnormality would seem either to cause or to arise concomitantly with language impairment in these patients.

Other findings on the developmental and possibly causal sequence of hearing impairments and other deficits in autism come from a review of studies on the relationship between mental health and auditory impairment in children. ${ }^{24}$ This review ${ }^{24}$ confirms the unusually high comorbidity of deafness and autism but reports that many researchers consider auditory impairment an unlikely etiological factor in autism. Other studies mentioned in the review ${ }^{25,26}$ for example-debate the possible etiological connections between auditory impairment and mental retardation when the two coexist in patients with autism. This particular area of the field is quite young. Further research into the developmental sequence of auditory impairments in autism is clearly necessary.

An ERP study by Bruneau and colleagues ${ }^{27}$ also found increased right hemisphere activation in response to sound among autism patients and found that the higher the verbal and communicative skills of the autistic patient, the higher the amplitude of the right hemispheric response. This study is one of a few correlating clinical and electrophysiological features in the auditory responses of patients with autism. However, the study's exclusive use of nonverbal auditory cues is problematic, given the link 
made to communicative skills. Unfortunately, the design choice renders the study's findings somewhat less useful to clinicians and researchers. Nonetheless, the group's approach and initial results may prove instructive for future studies.

A study by Bruneau and colleagues ${ }^{27}$ is also instructive in its recognition of the possible heterogeneity of language impairments among individuals with autism. Yet, Jarrold and colleagues ${ }^{28}$ have demonstrated that there was little heterogenentiy among language skills in patients with autism. However, a follow-up study by Kjelgaard and Tager-Flusberg ${ }^{29}$ has demonstrated significant heterogeneity among language skills, identifying impairment subgroups among patients with autism. While much work remains to be done in this area, it is clear that studies of, or built around, linguistic impairment in autism can no longer treat such impairment as uniform. Just as discussion of impairments of auditory perception has been oversimplified, linguistic impairment in autism has likewise been treated as a more uniform entity than it may well turn out to be.

Thus, studies of both speech and social auditory stimulus perception in autism have revealed impaired attentional orienting to these stimuli on one hand and atypical functional localization of this processing on the other. These results may hold important functional implications for individuals with autism, given their potential correlation with the social and linguistic deficits observed in the disorder.

\section{ENHANCED PERCEPTION OF PITCH AND MUSIC}

By contrast with impaired perception of social auditory cues, a growing body of studies has identified enhancements in auditory perception of pitch and music among autism patients. Older, cruder evidence of this enhancement, available since at least Blackstock's 1978 publication ${ }^{30}$ on autistic children's preference for musical over verbal acoustic stimuli, has been substantiated by many more recent findings. ${ }^{7,9,10,31,32}$ The observed aptitude for processing non-linguistic musical tones may relate to the restrictive activities and interests feature of autism, a category of symptoms that encompasses many autism patients' observed fascination with particular sensory stimuli. Enhanced melodic perception may also, some suggest, ${ }^{7,9,10}$ relate to specifically musical savant-like (though true savantism is rare) abilities of many children with autism.

Heaton and colleagues ${ }^{9,31}$ have recently published studies on enhancements in musical perception among patients with autism. A study by Heaton and col- leagues ${ }^{9}$ found that children with autism (with no prior musical training) could judge melodic pitch interval changes better than control children. Another study by Heaton and colleagues ${ }^{31}$ investigated the ability of children with autism to perceive affect in music, given their normal impairments in social affective perception. They found that children with autism can perceive affect on par with age- and intelligence-matched controls, as measured by their abilities to correctly identify affective connotations (happy or sad) of music in minor and major scales. These results suggest interesting therapeutic possibilities for harnessing autistic children's affective perceptions in the auditory domain to improve their interpersonal affective deficits. Precisely this sort of therapeutic application illustrates the clinical import of improved understanding of distinctions among auditory abnormalities.

Several studies have shown enhanced pitch discrimination in children with autism. Two studies by Heaton and colleagues ${ }^{9}$ and Heaton and colleagues ${ }^{10}$ have shown this enhanced ability in children with no musical background. Still another study by Heaton ${ }^{32}$ has shown enhanced pitch memory among children with autism. Bonnel and colleagues ${ }^{7}$ have further supported these findings in a study comparing the pitch discrimination abilities of high-functioning autistic individuals to normal controls in two signal discrimination tasks. None of the participants were musically trained. Subjects with autism showed a markedly increased sensitivity for changes in pitch in both the "same-different" and "high-low" pitch discrimination tasks relative to the control group. Bonnel and colleagues $^{7}$ relate these results to findings on enhanced visual discrimination among autistic patients, though the validity of this parallel is uncertain.

Generalized hypersensitivity to sound represents another type of enhanced non-linguistic auditory perception observed in many autistic patients. . $1,33,34^{2}$ Rosenhall and colleagues ${ }^{33}$ have reported an 18\% prevalence rate of hyperacusis (abnormally acute hearing due to increased sensory or neural sensitivity, characterized by intolerance for normal levels of sound) among subjects with autism versus none of the control subjects. ${ }^{33}$ In an ERP study, Gomot and colleagues ${ }^{8}$ found electrophysiological evidence for this feature, comparing the ERP responses with changes in sound frequency of pure tones among children with autism to those of normal controls. ${ }^{8}$ Specifically, they noted a shorter latency in the scalp current density mapping of mismatch negativity among autism patients. Though autism and control groups showed bilateral processing of sound, this abnormally early component noted among children 
with autism was restricted to the left frontal cortex. This suggests localization of their enhanced sensitivity to that region. Gomot and colleagues ${ }^{8}$ proposed a link between this finding and the classical hypothesis of frontal lobe abnormalities in autism. However, they also noted that ERPs may result from overlapping frequencies rather than from a single signal originating where the trace is recorded.

Many ERP studies similar to the investigation by Gomot and colleagues ${ }^{8}$ have been performed with widely varying results. Ferri and colleagues ${ }^{35}$ describe one widespread ERP finding of a decrease in the P300 wave among autism patients, the possible significance of which is discussed below. But when Kemner and colleagues $^{6}$ attempted to reproduce the results from all such studies, their only findings were those discussed earlier. Gomot and colleagues 8 proposed that the differences between their results and those of Kemner and colleagues ${ }^{6}$ are explained by the differences in stimuli-Kemner and colleagues ${ }^{6}$ used speech stimuli, Gomot and colleagues ${ }^{8}$ used non-linguistic tonesand subject pools-Kemner and colleagues ${ }^{6}$ tested high functioning autistic children only.

Given the vastly different results of similar studies of linguistic stimuli reviewed above, that former factorthe verbal nature of the auditory stimulus in the study Kemner and colleagues ${ }^{6}$ - may be particularly detrimental in this case. Indeed, as opposed to the impairment of auditory perception observed among autistic patients' response to linguistic and social stimuli, the body of studies on non-verbal stimuli has discovered a range of enhancements in autistic patients' perception of non-linguistic melodies and pure tones. This enhancement may relate to the restrictive and repetitive interest symptom cluster of autism, but this functional correlation remains to be proven. We return to this possibility in our discussion of the Weak Central Coherence theory in "Global versus Local Processing."

\section{IMPAIRMENTS IN NON-LINGUISTIC SOUND PROCESSING}

Studies of non-musical, non-linguistic auditory processing in patients with autism have generally found not enhanced but impaired perception relative to nonautistic peers. Thus, a simple dual model of impaired speech perception on one hand, and enhanced nonlinguistic acoustic perception on the other, does not hold. For example, Gage and colleagues ${ }^{36}$ and Gage and colleagues ${ }^{37}$ recently published magnetoencephalography (MEG) studies on deficiencies in right hemisphere processing of non-linguistic pure tones among autism patients. Both studies focused on the proportionality between the latency time of the
M100 brainwave and the frequency of a particular tone. Among control subjects, latency length increases as frequency increases but latency times shrink overall as a neurotypical individual matures. The first study by Gage and colleagues ${ }^{36}$ demonstrated a reduction in this latter age-dependent effect in the right hemisphere of autism patients, showing that latency times in that hemisphere of patients with autism do not shrink as much as they do in the right hemisphere of control subjects. Then Gage and colleagues ${ }^{37}$ demonstrated that the usual proportionality observed between frequency and latency is limited in the right hemisphere in autism patients, the right hemisphere showing a highly restricted range of latency times relative to the left. Both groups have proposed a relationship between their findings and those of $\mathrm{Pell}^{38}$ on the association of right hemisphere damage with deficiencies in decoding spectral features in sound. This possibility will be discussed later in the article. (It should be noted that the debate over hemispheric dominance in auditory processing in autism is ongoing, dating back to when Blackstock $^{30}$ reported right hemispheric dominance in both verbal and non-verbal acoustic processing in children with autism.) A possibly related finding on longer latency times comes from Rosenhall and colleagues, ${ }^{39}$ who reported greater latency times in auditory brainstem responses among patients with autism.

An MEG study by Oram Cardy and colleagues ${ }^{40}$ of the less-studied M50 wave found no difference between the M50 amplitudes measured in children with autism and those measured in control children in response to pure tones. Both groups showed greater amplitudes in the M50 compared with the M100 waves, whereas the normal adult group showed fardiminished M50 amplitudes relative to the M100. Oram Cardy and colleagues ${ }^{40}$ posited that a developmental shift in the relative importance of the M50 and M100 waves occurs during adolescence. However, because no adults with autism were studied, no definitive conclusions about auditory development in patients with autism can be drawn.

A different MEG study on tone processing in children with autism, this one by Tecchio and colleagues, ${ }^{41}$ examined neither the M100 nor the M50 waves. The focus of this study was the mismatch field (MMF) wave. The MMF wave is understood as an attention-independent marker of the detection of change in characteristics-in this case, change in frequency — of a repetitive sound and depends on the subject's memory trace of the preceding sound. The researchers found no MMF wave to be present in children with autism, as opposed to the robust wave present in control subjects. They understood this 
absence as evidence of an attention-independent deficiency in autism patients' ability to discriminate among the physical properties of two consecutively presented tones. This would seem to contradict findings $\mathrm{s}^{7,9,10,32}$ cited earlier on the increased perception of changes in pitch among patients with autism. One major difference, however, is that in this study, subjects were asked to identify frequency changes in a string of single, repetitive tones-to identify the $1,200 \mathrm{~Hz}$ tone among the otherwise similar 1,000 $\mathrm{Hz}$ tones-rather than pitch changes embedded in melodies. While the line between melody and sound is far from clear, studies like these are helping to functionally differentiate the two where the perceptions of individuals with autism are concerned.

Finally, the aforementioned study by Muller and colleagues ${ }^{19}$-discussed in the context of impairments in linguistic processing-describes overall reductions in auditory cortical activity during acoustic processing and reduced cerebellar activity in specifically non-verbal auditory processing. Whether and how these reductions in activity in specific brain areas relative to control subjects are compensated for by activity in other areas of the brain is unclear. If such compensation indeed exists, as Kemner and colleagues ${ }^{6}$ have hypothesized in the case of occipital cortical involvement in linguistic auditory processing, this reduction in activity may not, in fact, represent impaired function but, rather, abnormal functional localization. However, an alternate site of activity has yet to be shown. In the meantime, the evidence for this reduced activity in typically activated sites remains unconfirmed.

Thus, far from being characterized by a separation between impairments in linguistic processing, on one hand, and enhancements in non-linguistic processing, on the other, studies of abnormalities in auditory processing among autism patients reveal impairments in linguistic processing and both impairments and enhancements in non-linguistic processing.

Part of this distinction may be attributed to descriptive ambiguity in the field. For example, Rosenhall and colleagues ${ }^{28}$ discuss hyperacusis in the context of auditory deficits, rather than as a gain of function, as described here. Yet the directional heterogeneity-with major gains of function in some areas and major losses in others-among this body of findings as a whole seems too great to be explained away as an artifact of diction. The specificity to this directionality seems to lie in the details the brain waves studied, and the experimental design. A recent review by Bomba and Pang ${ }^{42}$ of the cortical auditory-evoked potentials in autism reveals the heterogeneity among this class of findings. While that review excludes findings on musical perception, and only cursorily addresses linguistic perception, it represents an excellent resource for those interested in the research on non-verbal, non-musical perception addressed in this section.

\section{GLOBAL VERSUS LOCAL PROCESSING}

One approach to making sense of the apparently discordant findings of both impairment and enhancement in non-linguistic auditory processing among autism patients has been to focus on distinctions between global and local auditory processing. For example, Mottron and colleagues ${ }^{43}$ found that while children with autism outperform their non-autistic peers in local processing abilities-namely, their recognition of single-note changes in melody-these children showed no improvement over peers in global processing skills, such as perception of changes in key of the entire melody or of alterations in melody contour. The basic theory is commonly traced back to Frith, ${ }^{44}$ who proposed that an apparent bias toward local processing over global processing among individuals with autism might help explain observations, such as enhanced pitch perception among autism patients. More recently, Frith and Happe ${ }^{45}$ described this abnormality not as enhanced local processing but as impaired global processing, which they termed weakened central coherence (WCC). The WCC theory states that deficient integration of local differences (eg, pitch) into a larger sonic whole enables the improved differentiation of local features observed among individuals with autism when compared with normal controls.

Concomitant with the question of enhanced local versus weakened global processing are study design questions of what precisely constitutes global or local processing. Foxton and colleagues ${ }^{46}$ object to studies like those of Mottron and colleagues, ${ }^{43}$ which use pitch contour as a measure of global processing. Such studies understand the processing of a succession of ascending and descending pitches to be a function of global processing, as opposed to the processing of any individual pitch or the comparison of two pitches, both of which types of processing would be local. Foxton and colleagues ${ }^{46}$ do not believe that sequencing a series of local processings should actually be understood as the simple addition of local processings that is, therefore, itself local as well. Foxton and colleagues ${ }^{46}$ were also unable to replicate the findings of Mottron and colleagues ${ }^{43}$ of enhanced local processing in their study. They, therefore, disputed not only Mottron and colleagues' ${ }^{46}$ analysis of their findings but also the findings themselves. 
Instead, Foxton and colleagues ${ }^{46}$ measured global processing as the combining of different local auditory features within a single sound, rather than as the sequencing of sounds that all differ by the same local feature. ${ }^{41}$ In contrast to the findings of Mottron and colleagues, ${ }^{43}$ using these measures of global versus local processing, Foxton and colleagues ${ }^{46}$ found not an enhancement in local processing but deficient global processing among patients with autism, a finding in line with the WCC theory. ${ }^{45}$ This latter form of the theory may also be supported by the finding in many ERP studies of a decreased P300 wave in autism patients, believed to indicate impaired ability to modify expectations in response to contextual cues. ${ }^{30}$

Whether the distinction between enhanced local processing and deficient global processing is a real one or merely an artifact of study design has yet to be shown. Nonetheless, the larger proposal that some abnormality in the balance of global and local processing may explain the non-linguistic auditory abnormalities in autism seems promising. Enhanced local processing could go far toward explaining the observed hypersensitivity to sound and enhanced pitch perception, along with the entire symptom cluster of repetitive and restrictive behaviors observed among individuals with autism. Deficient global processing, meanwhile, could explain functional reductions in higher-order auditory processing observed in autism patients, including the social and linguistic processing, that may relate to the other two symptom clusters in autism: linguistic and social impairment. As noted earlier, Gage and colleagues ${ }^{36}$ proposed that limitations in right hemispheric frequency M100 latency proportionality may relate to right hemispheric control over spectral decoding and perception of emotional contours of sound, either or both of which may be subsets of global processing, depending upon how that term is understood. ${ }^{31,32}$ Thus, the theory may go a long way toward explaining the variety of auditory abnormalities observed in autism and functionally relating those abnormalities to the symptoms of the disorder.

\section{CONCLUSION}

Studies of auditory abnormalities in autism are by no means a new frontier but technological advances and new interest have meant a resurgence of research interest in the field. While functional distinctions among findings in this area are poorly established to date, this review has suggested some general trends as a starting point for future analyses. Data on speech and social stimulus-specific auditory abnormalities appear, by and large, to indi- cate attentional orienting impairments and abnormal functional localization of linguistic processing among individuals with autism. Yet, a number of pitch and music-specific enhancements in perception have been noted among autism patients of all functioning levels. These gains of ability may relate more broadly to enhanced local processing abilities among autism patients, ${ }^{39,40}$ as hypothesized by Frith. ${ }^{44}$ Finally, a number of non-linguistic auditory processing impairments have also been noted, perhaps corresponding to impairments in global processing among this population.

Improved understanding of the nuances of auditory-processing abnormalities would greatly facilitate both treatment of patients with autism and further research on the disorder. As researchers and clinicians learn to better correlate specific abnormalities with specific symptoms (eg, impaired attentional orienting to speech with communication deficits), treatment and research will be able to more precisely target underlying etiologies.

Simultaneously, understanding the relationships among apparently contradictory findings, such as those of enhanced pitch perception and reduced right hemispheric frequency-latency proportionality, may illuminate larger, more pervasive cognitive patterns that cut across categories of symptomatology within the disorder. The distinction between global and local processing is only one such pattern yet proposed; what other myriad principles may be substantiated by, or emerge from, studies of auditory processing abnormalities in autism remains to be seen. CNS

\section{REFERENCES}

1. Diagnostic and Statistical Manual of Mental Disorders. 4th ed. text rev. Washington, DC: American Psychiatric Association; 2000.

2. Rogers SJ, Hepburn S, Wehner E. Parent reports of sensory symptoms in toddlers with autism and those with other developmental disorders. J Autism Dev Disord. 2003;33:631-642.

3. Dawson G, Meltzoff AN, Osterling J, Rinaldi J, Brown E. Children with autism fail to orient to naturally occurring social stimuli. J Autism Dev Disord. 1998;28:479-485.

4. Klin A. Young autistic children's listening preferences in regard to speech: a possible characterization of the symptom of social withdrawal. J Autism Dev Disord. 1991;21:29-42.

5. Ceponiene R, Lepisto T, Shestakova A, et al. Speech-sound-selective auditory impairment in children with autism: they can perceive but do not attend. Proc Natl Acad Sci U S A. 2003;100:5567-5572.

6. Kemner C, Verbaten MN, Cuperus JM, et al. Auditory event-related brain potentials in autistic children and three different control groups. Biol Psychiatry. 1995;38:150-165.

7. Bonnel A, Mottron L, Peretz I, et al. Enhanced pitch sensitivity in individuals with autism: a signal detection analysis. J Cogn Neurosci. 2003;15:226-235.

8. Gomot M, Giard MH, Adrien JL, Barthelemy C, Bruneau N. Hypersensitivity to acoustic change in children with autism: electrophysiological evidence of left frontal cortex dysfunctioning. Psychophysiology. 2002;39:577-584.

9. Heaton P, Pring L, Hermelin B. Musical processing in high functioning children with autism. Ann N Y Acad Sci. 2001;930:443-444.

10. Heaton P, Hermelin B, Pring L. Autism and pitch processing: a precursor for savant musical ability? Music Perception. 1998;15:291-305.

11. Ornitz EM. Autism at the interface between sensory and information processing. In: Dawson G, ed. Autism: Nature, Diagnosis, and Treatment. New York, NY: Guilford Press; 1989:174-207. 
12. Sinha Y, Silove N, Wheeler D, et al. Auditory integration training and other sound therapies for autism spectrum disorders. Cochrane Database Syst Rev. 2004;1: CD003681.

13. Siegal M, Blades M. Language and auditory processing in autism. Trends Cogn Sci. 2003; 7:378-380.

14. Auditory integration training and facilitated communication for autism. American Academy of Pediatrics. Committee on Children with Disabilities. Pediatrics. 1998;102(2 pt 1):431-433.

15. Mendelson MJ, Haith MM. The relation between audition and vision in the newborn. SRCD Monographs. 1976;41. (Serial No. 167.)

16. Dawson G, Toth K, Abbott R, et al. Early social attention impairments in autism: social orienting, joint attention, and attention to distress. Dev Psychol. 2004:40:271-283.

17. Courchesne E, Kilman BA, Galambos R, Lincoln AJ. Autism: processing of novel auditory information assessed by event-related brain potentials. Electroencephalogr Clin Neurophysiol. 1984;59:238-248.

18. Courchesne E, Lincoln AJ, Kilman BA, Galambos R. Event-related brain potential correlates of the processing of novel visual and auditory information in autism. J Autism Dev Disord. 1985;15:55-76.

19. Gervais H, Belin P, Boddaert N, et al. Abnormal cortical voice processing in autism. Nat Neurosci. 2004;7:801-802.

20. Muller RA, Behen ME, Rothermel RD, et al. Brain mapping of language and auditory perception in high-functioning autistic adults: a PET study. J Autism Dev Disord. 1999;29:19-31.

21. Boddaert N, Belin P, Chabane N, et al. Perception of complex sounds: abnormal pattern of cortical activation in autism. Am J Psychiatry. 2003;160:2057-2060.

22. Thivard L, Belin P, Zilbovicius M, Poline JB, Samson Y. A cortical region sensitive to auditory spectral motion. Neuroreport. 2000;11:2969-2972.

23. Samson Y, Belin P, Thivard L, et al. Auditory perception and language: functional imaging of speech sensitive auditory cortex [French]. Rev Neurol (Paris). 2001;157:837-846

24. Bailly D, Dechoulydelenclave MB, Lauwerier L. Hearing impairment and psychopathological disorders in children and adolescents. Review of the recent literature. Encephale. 2003;29(4 pt 1):329-337.

25. Gillberg C, Steffernburg S. Debate and argument: reply to AG Gordon: the evidence for a pathogenic role of peripheral hearing deficits in autism is extremely limited [discussion]. J Child Psychol Psychiatry. 1993;34:593-596.

26. Gordon AG. Debate and argument: interpretation of auditory impairment and markers for brain damage in autism. J Child Psychol Psychiatry. 1993;34:587-592.

27. Bruneau N, Bonnet-Brilhault F, Gomot M, Adrien JL, Barthelemy C. Cortical auditory processing and communication in children with autism: electrophysiologicaly/behavioral relations. Int J Psychophysiol. 2003;51:17-25.
28. Jarrold C, Boucher J, Russell J. Language profiles in children with autism: theoretical and methodological implications. Autism. 1997;1:57-76.

29. Kjelgaard M, Tager-Flusberg H. An investigation of language impairment in autism: Implications for genetic subgroups. Lang Cogn Process. 2001;16:287-308.

30. Blackstock, G. Cerebral asymmetry and the development of early infantile autism. J Autism and Childhood Schizophrenia. 1978;8:339-353.

31. Heaton P, Hermelin B, Pring L. Can children with autistic spectrum disorders perceive affect in music? An experimental investigation. Psychol Med. 1999;29:1405-1410.

32. Heaton P. Pitch memory, labelling and disembedding in autism. J Child Psychol Psychiatry. 2003;44:543-551.

33. Rosenhall U, Nordin V, Sandstrom M, Ahlsen G, Gillberg C. Autism and hearing loss. J Autism Dev Disord. 1999;29:349-357.

34. Chen L, Toth M. Fragile X mice develop sensory hyperreactivity to auditory stimuli. Neuroscience. 2001;103:1043-1050.

35. Ferri R, Elia M, Agarwal N, et al. The mismatch negativity and the P3a components of the auditory event-related potentials in autistic low-functioning subjects. Clin Neurophysiol. 2003;114:1671-1680.

36. Gage NM, Siegel B, Roberts TP. Cortical auditory system maturational abnormalities in children with autism disorder: an MEG investigation. Brain Res Dev Brain Res. 2003;144:201-209

37. Gage NM, Siegel B, Callen M, Roberts TP. Cortical sound processing in children with autism disorder: an MEG investigation. Neuroreport. 2003;14:2047-2051.

38. Pell MD. Fundamental frequency encoding of linguistic and emotional prosody by right hemisphere-damaged speakers. Brain Lang. 1999;69:161-192.

39. Rosenhall U, Nordin V, Brantberg K, Gillberg C. Autism and auditory brain stem responses. Ear Hear. 2003;24:206-214.

40. Oram Cardy JE, Ferrari P, et al. Prominence of M50 auditory evoked response over M100 in childhood and autism. Neuroreport. 2004;15:1867-1870.

41. Tecchio F, Benassi F, Zappasodi F, et al. Auditory sensory processing in autism: a magnetoencephalographic study. Biol Psychiatry. 2003;54:647-654.

42. Bomba MD, Pang EW. Cortical auditory evoked potentials in autism: a review. Int J Psychophysiol. 2004;53:161-169.

43. Mottron L, Peretz I, Menard E. Local and global processing of music in high-functioning persons with autism: beyond central coherence? J Child Psychol Psychiatry. 2000;41:1057-1065.

44. Frith U. Autism: Explaining the Enigma. Oxford, England: Blackwell Press; 1989.

45. Frith U, Happe F. Autism: beyond 'theory of mind'. Cognition. 1994;50:115-1132.

46. Foxton JM, Stewart ME, Barnard L, et al. Absence of auditory 'global interference' in autism. Brain. 2003;126:2703-2709. 